\title{
Process for the determination of thickness of polymeric microchannels for microfluidic applications
}

\begin{abstract}
We have developed a technique of fabricating device channels for microfludics system by using high performance epoxy polymeric based dry film resists (DFRs). We will use the fabrication method originated in silicon microelectronics fabrication industry. This is because the interest in this industry has made the current microfluidic devices fabricated not only from silicon substrate but also to a range of polymers and glasses. Our observations are made on the effect of the thickness before and after curing and bonding mechanism of DFR on glass substrate. Therefore the thickness of the channels is recorded. The outcomes of the bonding procedure are captured. These channels are patterned and sandwiched in between two glass substrates. They can be used for handling continuous fluid flow and particle. In our advance, the channel was formed for dielectrophoretic (DEP) colloidal particle separation systems.
\end{abstract}

Keyword: Colloidal particles; Dry film resist; Microfluidic; Separation; Thickness 